\title{
Targeted Treatment of Chronic Lymphocytic Leukemia: Clinical Utility of Acalabrutinib
}

\author{
Candida Vitale (D) \\ Jamie Lynn Gibbons ${ }^{2}$ \\ Alessandra Ferrajoli ${ }^{2}$ \\ 'Department of Molecular Biotechnology \\ and Health Sciences, University of Torino \\ and University Division of Hematology, \\ A.O.U. Città della Salute e della Scienza di \\ Torino, Torino, Italy; ${ }^{2}$ Department of \\ Leukemia, The University of Texas MD \\ Anderson Cancer Center, Houston, \\ TX, USA
}

\begin{abstract}
In chronic lymphocytic leukemia (CLL), a deeper understanding of the disease biology led over the last decade to the development and clinical use of different targeted drugs, including Bruton tyrosine kinase (BTK) inhibitors. The first BTK inhibitor approved for clinical use is ibrutinib, which showed excellent efficacy and good tolerability. More recently, the interest is growing for novel more selective BTK inhibitors that may reduce the off-target effects of the drug, thus minimizing side effects and subsequent treatment interruptions or discontinuations. Acalabrutinib is an orally administered irreversible BTK inhibitor, characterized by the lack of inhibition towards other kinases. In this review, we present the most recent data from clinical trials on the clinical efficacy of acalabrutinib and acalabrutinib-based combinations for the treatment of patients with relapsed/refractory and treatment-naïve CLL. We delineate the safety profile of the drug, describe side effects of interest and discuss the clinical management of patients receiving acalabrutinib. Due to its efficacy and the favorable safety profile, acalabrutinib has emerged as a viable therapy option in the current landscape of multiple approved treatments for CLL.
\end{abstract}

Keywords: chronic lymphocytic leukemia, acalabrutinib, Bruton tyrosine kinase inhibitor

\section{Introduction}

Chronic lymphocytic leukemia (CLL) is a B-cell lymphoproliferative disease that accounts for $1.1 \%$ of all cancer diagnosis in the US. ${ }^{1}$ Historically, the therapeutic armamentarium against CLL consisted of chemotherapy agents, used alone or in combination with the anti-CD20 monoclonal antibody rituximab. However, a deeper understanding of the disease biology led over the last decade to the development and clinical use of different drugs, specifically directing their effects to targets that are essential for tumor B-cell survival. Bruton tyrosine kinase (BTK), a kinase predominantly expressed in hematopoietic cells, was identified as a fundamental component of the B-cell receptor (BCR) signaling pathway, whose activity is needed to ensure the survival and proliferation of malignant B cells. ${ }^{2}$ BTK demonstrated to be a key therapeutic target in B-cell tumors, and especially in CLL. ${ }^{2}$ The first BTK inhibitor which was approved for clinical use in the setting of CLL is ibrutinib. Long-term results from the pivotal Phase III studies RESONATE and RESONATE-2 confirmed excellent efficacy. ${ }^{3,4}$ More recently, phase III trials have demonstrated the superiority of ibrutinib treatment as compared to chemo-immunotherapy in different groups of previously untreated patients with CLL. ${ }^{5-7}$ Ibrutinib is given as continuous treatment at a dose of $420 \mathrm{mg} /$ day until disease progression or unacceptable toxicity. The treatment with ibrutinib is generally
Correspondence: Alessandra Ferrajol Department of Leukemia, Unit 428, The University of Texas MD Anderson Cancer Center, 1515 Holcombe Boulevard, Houston, TX, 77030, USA

Tel + I $7 / 3$ 792-2063

Fax $+1713792-9616$

Email aferrajo@mdanderson.org 
considered well tolerated, and patients with comorbidities can be most of the time safely treated with this drug. Nevertheless, the cumulative experience from multiple trials, together with data from the real-world practice, have highlighted the unique side effect profile of this drug, which specifically include atrial fibrillation, hypertension and bleeding episodes. ${ }^{8-10}$ It needs to be highlighted that ibrutinib-induced toxicities may include severe events, but also low-grade persistent side effects. All these adverse events (AEs) may lead to treatment interruption or definitive discontinuation and may represent an issue for patients' treatment compliance and eventually for efficacy. ${ }^{11}$ Translational research efforts have been carried out with the aim of understanding the biological basis of these ibrutinib-induced specific toxicities. It has been shown that the off-target effects of the drug, such as sustained ibrutinib-induced inhibition of other kinases (eg, Tec, EGFR, ITK), may be responsible for some of the toxicities observed. ${ }^{12}$ From these observations stemmed the quest for novel more selective BTK inhibitors that may improve the tolerability of the treatment while maintaining the efficacy of the first-in-class drug.

Besides ibrutinib, other covalent BTK inhibitors have been clinically evaluated for the treatment of patients with CLL, such as acalabrutinib (ACP-196), zanubrutinib (BGB-3111), spebrutinib (CC-292) and tirabrutinib (ONO-4059/GS-4059). Additionally, novel generation BTK inhibitors that exploit their inhibitory mechanism in a reversible and non-covalent manner are also in development (ie pirtobrutinib (LOXO-305), MK1026 (formerly ARQ-531) and CG806). This review will specifically focus on the clinical utility - in the setting of CLL - of acalabrutinib, being the first next-generation BTK inhibitor that was approved for the clinical use, and for which data are more mature to date.

\section{Acalabrutinib: Mechanism of Action, Preclinical Data and Dose Scheduling Evaluation}

Acalabrutinib is an orally administered irreversible BTK inhibitor that forms a covalent bond with C481 in the ATPbinding pocket (Figure 1). As compared to ibrutinib, acalabrutinib is highly selective and characterized by the lack of inhibition towards other kinases. ${ }^{13-15}$ In vitro, the selectivity of acalabrutinib appears to be comparable to that of tirabrutinib and higher compared to ibrutinib, zanubrutinib and spebrutinib. ${ }^{16}$ This improved selectivity should limit the occurrence of ibrutinib-related off-target AEs. For example, it has been hypothesized that a reduced inhibition of Src family kinases by acalabrutinib - compared to ibrutinib - may ameliorate the platelet dysfunction observed in patients treated with other BTK inhibitors. $^{13,17,18}$

Besides the reduction of side effects, the aim of acalabrutinib is also that of maintaining the excellent therapeutic results of the first-in-class compound. In the preclinical in vitro setting, acalabrutinib shows a dosedependent BCR inhibition in primary CLL cells. ${ }^{13}$ The molecular and biological effects of ibrutinib and acalabrutinib appear similar in terms of intracellular downstream pathways events, cytotoxicity, chemokine production and cell migration. ${ }^{19}$ Interestingly, there were also some differences noted: in vitro studies showed that the drug-induced cell death rate is slightly higher for ibrutinib than acalabrutinib, possibly due to the off-target effects of ibrutinib. Conversely, the two drugs have a differential effect on healthy $\mathrm{T}$ lymphocytes, ibrutinib showing more pronounced off-target effects on Src-family kinases. However, when the in vivo effects of ibrutinib and acalabrutinib on $\mathrm{T}$ cells were evaluated, both drugs showed the ability of downmodulating immune-suppressive molecules such as PD-1 and CTLA-4. ${ }^{20}$ Additionally, in two distinct murine models of CLL, acalabrutinib confirmed its potent BTK inhibition and selectivity in vivo, and showed a significant inhibition of CLL cell proliferation, together with tumor burden reduction and survival increase compared to mice receiving vehicle. ${ }^{14}$

Correlative studies also explored the combinatorial activity of BTK inhibitors: it has been postulated that due to the off-target inhibition toward other kinases such as ITK or Tec, ibrutinib may negatively interact with the main mechanisms of action of anti-CD20 monoclonal antibodies, such as antibody-dependent cellular cytotoxicity and antibody-dependent phagocytosis. $^{21-23}$ Golay et al reported that acalabrutinib does not seem to affect the mechanisms of action of anti-CD20 monoclonal antibodies in vitro, possibly due to its increased specificity. ${ }^{24}$

Other acalabrutinib-containing drug combinations have also been assessed in vitro. For example, Patel et al showed that the Bcl-2 inhibitor venetoclax exerts increased cytotoxicity on CLL cells collected from acalabrutinib-treated patients compared to pretreatment samples and that in in vitro-treated CLL samples, the combination of acalabrutinib and venetoclax induces higher rates of cell death compared to both drugs used as single agents. ${ }^{25}$ 


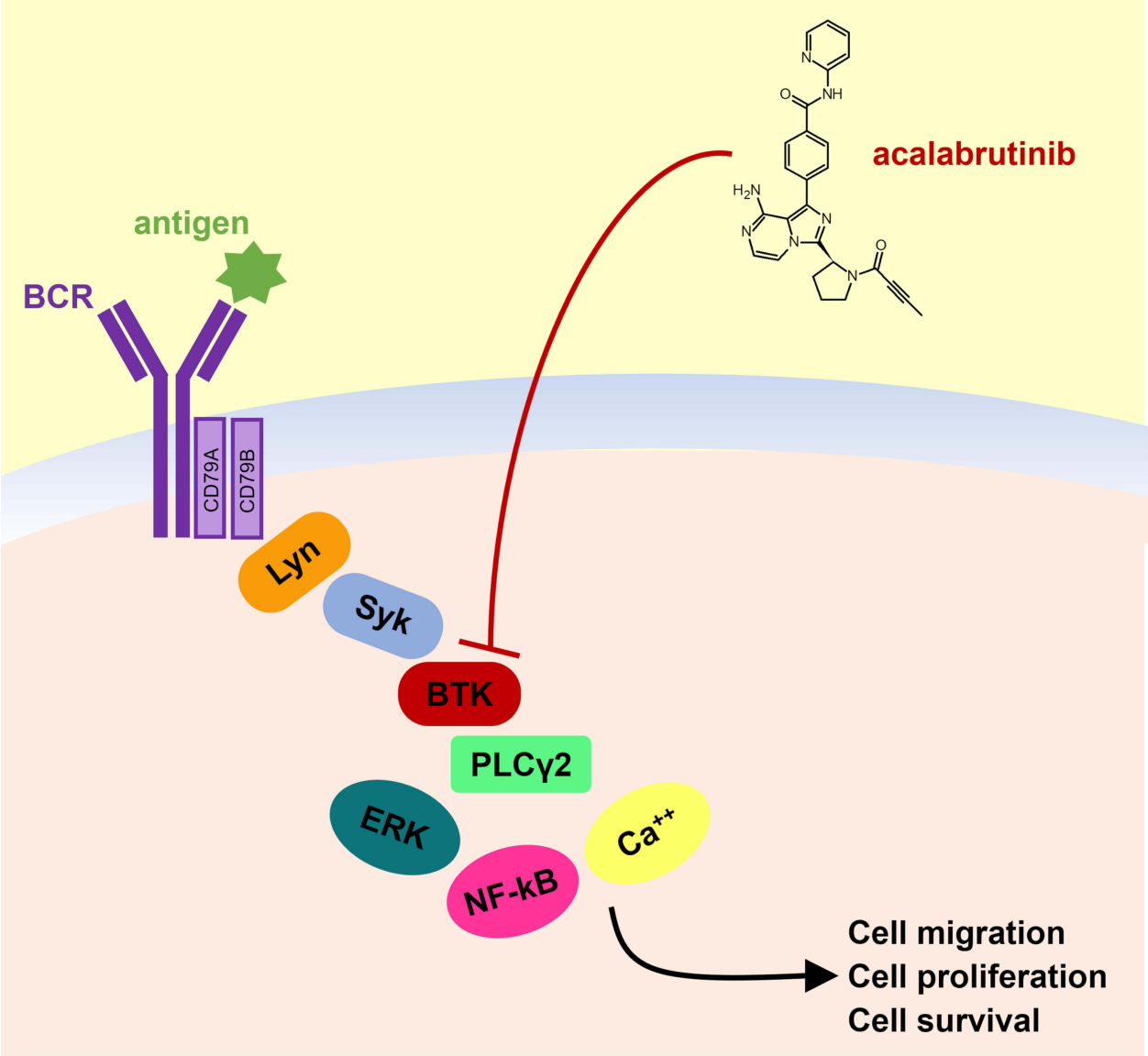

Figure I Acalabrutinib structure and mechanism of action.

Moreover, a possible therapeutic advantage of the combination of acalabrutinib and venetoclax combination was confirmed in a TCL-1 adoptive transfer mouse model. ${ }^{25}$

BTK occupancy by acalabrutinib has been measured in clinical trials. In the phase I/II study evaluating acalabrutinib in patients with CLL, all tested doses (ie $100 \mathrm{mg}, 175 \mathrm{mg}$, $250 \mathrm{mg}$ and $400 \mathrm{mg}$ ) achieved a complete BTK occupancy (99-100\%) 4 hours after dosing on day 8 (steady-state). ${ }^{13}$ However, the median BTK occupancy before next-dose administration obtained with once-daily dosing ranged from $87 \%$ to $95 \%$, whereas the $100 \mathrm{mg}$ twice daily dosing schedule showed optimal before-dosing BTK inhibition (97\%). As a comparison, the approved $420 \mathrm{mg}$ daily dose of ibrutinib achieves a BTK occupancy of approximately 90\% 4 hours after dosing on day 8 , and of approximately $80 \%$ before next-dose administration. ${ }^{26}$ The $100 \mathrm{mg}$ twice daily dosing was therefore chosen as the preferred dose for further acalabrutinib studies and for use in the clinical practice, the drug being generally given continuously until disease progression or unacceptable toxicity. Of note, in a recent study by Sun et al, the twice daily $100 \mathrm{mg}$ dose was confirmed to be able to achieve significantly higher BTK occupancy than the $200 \mathrm{mg}$ daily dose at different time points, although with a difference decreasing over time. ${ }^{27}$ Also, the BTK inhibition exerted in the lymph nodes - the tissue compartment where BCR activation and CLL cell proliferation mostly occur in CLL - was more potent in patients treated with the twice daily $100 \mathrm{mg}$ dose compared to the $200 \mathrm{mg}$ daily dose. ${ }^{27}$

\section{Clinical Efficacy of Acalabrutinib in CLL Acalabrutinib Monotherapy for Relapsed or Refractory Patients}

The ACE-CL-001 study (NCT02029443) is a phase I/II multicenter clinical trial, which enrolled patients with relapsed CLL and whose results were first published in $2016 .{ }^{13}$ In the dose-escalation phase, patients were treated with acalabrutinib at a dose of $100 \mathrm{mg}, 175 \mathrm{mg}, 250 \mathrm{mg}$ or $400 \mathrm{mg}$ once daily, whereas in the Phase II portion of the 
study, the drug was given $100 \mathrm{mg}$ twice daily. Sixty-one patients were treated, with a median age of 62 years and a median of 3 prior treatment lines (range, 1-13). Thirty-one percent of the patients had a del(17p) and 75\% had unmutated IGHV. After a relatively short median follow-up of 14.3 months, overall response rate (ORR) was $95 \%$ and only two events of progression or death were noted (one each). In the most recent update of the study, which reported on a population of 134 patients, at a median follow-up of 41 months, the high ORR was confirmed (94\%), regardless of genomic characteristics or complex karyotype. ${ }^{28}$ Fifty-six percent of the patients remained on treatment, the median progression-free survival (PFS) was not reached and the estimated 45 -month PFS was $62 \%$.

The multicenter randomized phase III ASCEND trial (ACE-CL-309, NCT02970318) treated 310 patients with relapsed/refractory CLL with acalabrutinib monotherapy $(n=155)$ or investigator's choice (idelalisib plus rituximab, $\mathrm{n}=119$, or bendamustine plus rituximab, $\mathrm{n}=36$, both regimens given at standard doses/schedule). ${ }^{29}$ Patients had a median of 2 prior therapies (range, 1-10), but prior Bcl-2 or BTK/PI3K inhibitors were not allowed. ORR was $81 \%$ and $75 \%$ for acalabrutinib and investigator's choice arms, respectively. At a median follow-up of 16 months, median PFS was significantly longer with acalabrutinib monotherapy (not reached) versus the investigator's choice cohort (16.5 months). The estimated 1-year PFS was $88 \%$ and $68 \%$ for acalabrutinib and investigator's choice arms, respectively.

Acalabrutinib was directly compared to ibrutinib in the phase III non-inferiority ELEVATE-RR trial (ACE-CL006, NCT02477696), which randomized 533 patients with previously treated CLL, carrying $\operatorname{del}(17 \mathrm{p})$ and/or del (11q). ${ }^{30}$ The ORR was $81 \%$ for acalabrutinib $(n=268)$ and $77 \%$ for ibrutinib $(n=265)$. At a median follow-up of 41 months, median PFS was 38.4 months for both treatment arms, and the prespecified criterion for non-inferiority was met. Median overall survival (OS) was not reached in either group, and median time-to-next treatment was similar between treatment arms.

\section{Acalabrutinib in Association with Obinutuzumab for Relapsed or Refractory Patients}

Although in previous trial experiences the addition of rituximab to ibrutinib did not result in significant clinical improvements, ${ }^{5,31}$ several studies assessed the efficacy of the combination of acalabrutinib with the anti-CD20 monoclonal antibody obinutuzumab, a type II humanized antibody which was specifically glycoengineered to optimize antibody-dependent cellular cytotoxicity.

The single-center phase Ib/II study ACE-CL-003 (NCT02296918) enrolled both treatment-naïve and relapsed/refractory patients with CLL. ${ }^{32}$ In the relapsed/ refractory cohort, the patients $(n=26)$ had a median age of 63 years and a median number of prior therapies of 1 (range, 1-9). Patients received acalabrutinib and obinutuzumab (100 mg on cycle 1 day 1; $900 \mathrm{mg}$ on cycle 1 day 2; $1000 \mathrm{mg}$ on cycle 1 day 8 and 15, and on day 1 of cycles 2-6), obtaining an ORR of $92 \%$. At a median follow-up 42 months, median PFS was not reached and the 3-year PFS rate was $88 \%$. Based on the scarcity of data available to date, it is not possible to determine whether the addition of obinutuzumab may improve the efficacy of acalabrutinib for the treatment of CLL in the relapsed/ refractory setting.

\section{Acalabrutinib as Frontline Therapy}

The phase I/II ACE-CL-001 trial also evaluated the efficacy and safety of acalabrutinib monotherapy in a cohort of 99 previously untreated patients with CLL, who declined treatment with chemo-immunotherapy or who had comorbidities that precluded it. ${ }^{33}$ The median age was 64 years, $62 \%$ of the patients had unmutated IGHV and $18 \%$ had TP53 aberrations. Acalabrutinib was initially given at a dose of $200 \mathrm{mg}$ once daily or $100 \mathrm{mg}$ twice daily, until progression or intolerance, but a protocol amendment later converted all patients to the twice daily dose. ORR was $97 \%$, with similar outcomes among all prognostic subgroups. After a median follow-up of 53 months, median PFS was not reached and the estimated 4-year PFS rate was $96 \%$ ( $82 \%$ in patients with del( $17 \mathrm{p})$ and/or mutated TP53, and $91 \%$ in patients with complex karyotype).

The above-mentioned ACE-CL-003 phase Ib/II study, which evaluated the association of acalabrutinib with obinutuzumab, enrolled 19 treatment-naïve patients, with a median age of 61 years. ${ }^{32}$ Fifty-three $\%$ of patients had unmutated IGHV, $22 \%$ had $\operatorname{del}(17 \mathrm{p}), 28 \%$ had a TP53 mutation and $42 \%$ had a complex karyotype. The ORR was $95 \%$ and at the median follow-up of 39 months, PFS rate was $94.4 \%$.

The phase III ELEVATE-TN study (ACE-CL-007, NCT02475681) enrolled patients with previously untreated CLL who were aged 65 years or older, or 18- 
65 years and who had a creatinine clearance of 30-69 mL/ min or a Cumulative Illness Rating Scale for Geriatrics (CIRS-G) score $>6 .{ }^{34}$ Five hundred thirty-five patients were randomly assigned to receive 1) acalabrutinib monotherapy $(n=179)$, or 2$)$ acalabrutinib and obinutuzumab (100 mg on cycle 2 day 1; $900 \mathrm{mg}$ on cycle 2 day 2; $1000 \mathrm{mg}$ on cycle 2 day 8 and 15, and on day 1 of cycles $3-7)(\mathrm{n}=179)$, or 3$)$ obinutuzumab (100 mg on cycle 1 day 1; $900 \mathrm{mg}$ on cycle 1 day 2; $1000 \mathrm{mg}$ on cycle 1 day 8 and 15 , and on day 1 of cycles $2-6)$ and chlorambucil $(0.5 \mathrm{mg} /$ $\mathrm{kg}$ on days 1 and 15 of cycles $1-6)(\mathrm{n}=177)$. Regarding biological disease characteristics, $9 \%$ of the patients had a $\operatorname{del}(17 \mathrm{p}), 18 \%$ had a del(11q), 63\% had unmutated IGHV and $17 \%$ had a complex karyotype. The best ORR was $94 \%, 86 \%$ and $79 \%$ in patients treated with acalabrutinibobinutuzumab, acalabrutinib monotherapy and obinutuzumab-chlorambucil, respectively. At a median follow-up of 28.3 months, patients treated with acalabrutinib-containing regimens had a longer median PFS compared with obinutuzumab-chlorambucil (not reached vs 28.3 months). PFS improvements were consistently observed in main patient subgroups. The estimated 2-year PFS was 93\%, 87\% and $47 \%$ in patients treated with acalabrutinib-obinutuzumab, acalabrutinib monotherapy and obinutuzumab-chlorambucil, respectively. The study was not powered to detect a PFS difference in patients treated on the two acalabrutinibcontaining arms, but in a recent post-hoc analysis, a trend was reported toward a longer PFS in patients who received acalabrutinib-obinutuzumab versus acalabrutinib monotherapy, with a 4-year estimated PFS of $87 \%$ and $78 \%$, respectively. ${ }^{35}$ The median OS was not reached in any treatment cohort, and no significant differences in terms of OS were observed among groups at this follow-up.

Together with the ASCEND trial, the ELEVATE-TN trial led - in November 2019 - to the approval of acalabrutinib for the treatment of patients with CLL in the United States. One year later, the European Union marketing authorization was issued. ${ }^{36}$ Acalabrutinib, with or without obinutuzumab, is currently indicated by international guidelines among the preferred frontline regimens for CLL, but the presence of multiple highly effective options poses an indisputable challenge for the clinicians. In the absence of head-to-head comparison, data from a recent network meta-analyses might be of help. ${ }^{37}$ Results from 8 clinical trials enrolling fludarabine-ineligible patients were analyzed, and the 5-year PFS rate for acalabrutinib and obinutuzumab treatment was higher than for ibrutinib, but not higher than for single agent acalabrutinib, ibrutinib and obinutuzumab, ibrutinib and rituximab, or venetoclax and obinutuzumab.

Relevant clinical trials of acalabrutinib for the treatment of patients with CLL are summarized in Table 1.

\section{Acalabrutinib for the Treatment of Ibrutinib-Intolerant Patients}

The phase I/II ACE-CL-001 study included a cohort of 33 patients who discontinued ibrutinib due to severe AEs. ${ }^{38}$ The median age of these patients was 64 years and median number of prior therapies was 4 (range, 2-13). Unfavorable prognostic factors were common: $38 \%$ of the patients had $\operatorname{del}(17 \mathrm{p}), 22 \%$ had $\operatorname{del}(11 \mathrm{q})$ and $81 \%$ had unmutated IGHV. The ORR was $76 \%$. The treatment was well tolerated, and at a median follow-up of 19 months, $70 \%$ of the patients remained on acalabrutinib therapy and $9 \%$ discontinued due to an AE. Median PFS was not reached, 1-year and 2-year PFS were $83.4 \%$ and $75 \%$, respectively. Of interest, among 61 ibrutinib-related AEs that were previously reported, $72 \%$ did not recur and $13 \%$ recurred at a lower grade during acalabrutinib therapy.

A phase II study to specifically assess the efficacy and tolerability of acalabrutinib in a larger cohort of ibrutinibintolerant CLL patients (ACE-CL-208, NCT02717611) is currently ongoing, and only preliminary results have been presented so far. $^{39}$

\section{Safety and Tolerability of Acalabrutinib in CLL}

As the efficacy of acalabrutinib has shown promising results, an in-depth understanding of its safety and tolerability is essential to define its place in the treatment of patients with CLL. In a pooled analysis of clinical trials with acalabrutinib monotherapy that included 1040 patients with median duration of exposure at 24.6 months, the most common AEs of any grade reported with acalabrutinib therapy were headache $(n=393,38 \%)$, diarrhea $(\mathrm{n}=382,37 \%)$, upper respiratory tract infection $(\mathrm{n}=229$, $22 \%)$, contusion $(n=226,22 \%)$, nausea $(n=226,22 \%)$, fatigue $(n=222,21 \%)$ and cough $(n=218,21 \%) .{ }^{40}$ For each AE, over $90 \%$ of those reported were grade 1 or 2 . For AEs of grade 3 or higher, the three most common noted were neutropenia $(n=116,11.2 \%)$, anemia $(n=81$, $7.85 \%)$ and pneumonia $(n=53,5.1 \%)$. Of the reported serious AEs, pneumonia was the most frequent $(n=51)$. In addition, it was the most frequent fatal $\mathrm{AE}$ reported 
Table I Relevant Clinical Trials of Acalabrutinib for the Treatment of Patients with CLL

\begin{tabular}{|c|c|c|c|c|}
\hline Trial & $\begin{array}{l}\text { Study Design/Agents Number of } \\
\text { Patients (on Acalabrutinib Arms) }\end{array}$ & $\begin{array}{l}\text { Median } \\
\text { Follow-Up }\end{array}$ & Results & AEs (\% of Patients) \\
\hline $\begin{array}{l}\text { ACE-CL-00I } \\
\text { Phase Ib/II } \\
\text { NCT02029443 }\end{array}$ & $\begin{array}{l}\text { Multicenter, open-label and dose-escalation } \\
\text { study of acalabrutinib } \\
\text { I34 RR }\end{array}$ & 41 months & $\begin{array}{l}\text { ORR, } 94 \% \\
45 \text {-month PFS, } 62 \% \\
\text { Median PFS, not reached }\end{array}$ & $\begin{array}{l}\text { Any grade } \\
\text { Diarrhea (52\%) } \\
\text { Headache }(51 \%) \\
\text { Hypertension (22\%) } \\
\text { Atrial Fibrillation (7\%) } \\
\text { Major Bleeding (5\%) } \\
\text { Grade } \geq 3 \\
\text { Neutropenia }(14 \%) \\
\text { Pneumonia (I } 1 \%) \\
\text { Hypertension }(7 \%)\end{array}$ \\
\hline $\begin{array}{l}\text { ACE-CL-00I } \\
\text { Phase Ib/II } \\
\text { NCT02029443 }\end{array}$ & $\begin{array}{l}\text { Multicenter, open-label and dose-escalation } \\
\text { study of acalabrutinib } \\
99 \mathrm{TN}\end{array}$ & 53 months & $\begin{array}{l}\text { ORR, } 97 \% \\
\text { Median DOR, not reached }\end{array}$ & $\begin{array}{l}\text { Grade } \geq \mathbf{3} \\
\text { Infection (I5\%) } \\
\text { Hypertension (II\%) } \\
\text { Bleeding events (3\%) } \\
\text { Atrial fibrillation (2\%) }\end{array}$ \\
\hline $\begin{array}{l}\text { ACE-CL-00I } \\
\text { Phase Ib/II } \\
\text { NCT02029443 }\end{array}$ & $\begin{array}{l}\text { Multicenter, open-label and dose-escalation } \\
\text { study of acalabrutinib } \\
\text { (ibrutinib-Intolerant cohort) } \\
33 \text { RR }\end{array}$ & 19 months & $\begin{array}{l}\text { ORR, } 76 \% \\
\text { 24-month PFS, } 75 \% \\
\text { Median PFS, not reached }\end{array}$ & $\begin{array}{l}\text { Any grade: } \\
\text { Diarrhea (58\%) } \\
\text { Headache (39\%) } \\
\text { Cough (33\%) } \\
\text { Grade } \geq 3 \text { : } \\
\text { Neutropenia (12\%) } \\
\text { Thrombocytopenia (9\%) }\end{array}$ \\
\hline $\begin{array}{l}\text { ACE-CL-208 } \\
\text { Phase II } \\
\text { NCT027I76II }\end{array}$ & $\begin{array}{l}\text { Multicenter, open-label study of } \\
\text { acalabrutinib in subjects who are intolerant } \\
\text { of ibrutinib } \\
60 \text { RR }\end{array}$ & 19 months & $\begin{array}{l}\text { ORR, } 77 \% \\
21 \text {-month PFS, } 76 \% \\
\text { Median PFS, not reached }\end{array}$ & $\begin{array}{l}\text { Any grade: } \\
\text { Diarrhea }(48 \%) \\
\text { Headache }(40 \%) \\
\text { Contusion }(35 \%) \\
\text { Dizziness }(32 \%)\end{array}$ \\
\hline $\begin{array}{l}\text { ACE-CL-003 } \\
\text { Phase Ib/II } \\
\text { NCT02296918 }\end{array}$ & $\begin{array}{l}\text { Study of acalabrutinib in combination with } \\
\text { obinutuzumab } \\
19 \mathrm{TN} \\
26 \mathrm{RR}\end{array}$ & 42 months & $\begin{array}{l}\text { ORR TN, } 95 \% \\
\text { ORR RR, } 92 \% \\
36-\text { month PFS TN, } 94 \% \\
\text { 36-month PFS RR, } 88 \% \\
\text { Median PFS, not reached } \\
\text { (TN, RR) }\end{array}$ & $\begin{array}{l}\text { Any grade: } \\
\text { Headache (56\%) } \\
\text { Grade } \geq 3 \text { : } \\
\text { Neutropenia (24\%) } \\
\text { Syncope (II\%) } \\
\text { Weight gain (9\%) } \\
\text { Hypertension (7\%) }\end{array}$ \\
\hline $\begin{array}{l}\text { ASCEND } \\
\text { ACE-CL-309 } \\
\text { Phase III } \\
\text { NCT02970318 }\end{array}$ & $\begin{array}{l}\text { Randomized, multicenter, open-label study } \\
\text { of acalabrutinib vs investigator's choice } \\
\text { (idelalisib plus rituximab or bendamustine } \\
\text { plus rituximab) } \\
\text { I55 RR }\end{array}$ & 22 months & $\begin{array}{l}\text { ORR, } 80 \% \\
\text { ORR + PRL, } 92 \% \\
\text { I8-month PFS, } 82 \% \\
\text { Median PFS, not reached }\end{array}$ & $\begin{array}{l}\text { Any grade: } \\
\text { Headache (22\%) } \\
\text { Diarrhea }(21 \%) \\
\text { Neutropenia }(20 \%) \\
\text { Grade } \geq 3 \text { : } \\
\text { Neutropenia (I7\%) } \\
\text { Anemia }(12 \%)\end{array}$ \\
\hline
\end{tabular}

(Continued) 
Table I (Continued).

\begin{tabular}{|c|c|c|c|c|}
\hline Trial & $\begin{array}{l}\text { Study Design/Agents Number of } \\
\text { Patients (on Acalabrutinib Arms) }\end{array}$ & $\begin{array}{l}\text { Median } \\
\text { Follow-Up }\end{array}$ & Results & AEs (\% of Patients) \\
\hline $\begin{array}{l}\text { ELEVATE-RR } \\
\text { ACE-CL-006 } \\
\text { Phase III } \\
\text { NCT02477696 }\end{array}$ & $\begin{array}{l}\text { Randomized, multicenter, open-label, non- } \\
\text { inferiority study of acalabrutinib vs } \\
\text { ibrutinib } \\
268 \text { RR }\end{array}$ & $4 I$ months & $\begin{array}{l}\text { ORR, } 81 \% \\
\text { Median PFS, } 38.4 \text { months }\end{array}$ & $\begin{array}{l}\text { Any grade: } \\
\text { Diarrhea (35\%) } \\
\text { Headache (35\%) } \\
\text { Cough (29\%) } \\
\text { URI (27\%) } \\
\text { Pyrexia (23\%) } \\
\text { Hypertension (9\%) } \\
\text { Grade } \geq 3 \text { : } \\
\text { Neutropenia (20\%) } \\
\text { Anemia (I2\%) } \\
\text { Pneumonia (II\%) } \\
\text { Thrombocytopenia (10\%) } \\
\text { Atrial Fibrillation (5\%) }\end{array}$ \\
\hline $\begin{array}{l}\text { ELEVATE-TN } \\
\text { Phase III } \\
\text { ACE-CL-007 } \\
\text { NCT0247568I }\end{array}$ & $\begin{array}{l}\text { Randomized, multicenter, open-label study } \\
\text { of obinutuzumab plus chlorambucil vs } \\
\text { acalabrutinib plus obinutuzumab vs } \\
\text { acalabrutinib monotherapy } \\
\text { I79 TN }(A+O) \\
\text { I79 TN (A) }\end{array}$ & 29.8 months & $\begin{array}{l}\text { ORR A+O, } 94 \% \\
\text { ORR A, } 86 \% \\
24-\text { month PFS A+O, 93\% } \\
\text { 24-month PFS A, 87\% } \\
\text { Median PFS, not reached } \\
(A+O, A)\end{array}$ & $\begin{array}{l}\text { Any grade }(\mathbf{A}+\mathbf{O}, \mathbf{A}) \text { : } \\
\text { Headache }(40 \%, 37 \%) \\
\text { Diarrhea }(39 \%, 35 \%) \\
\text { Neutropenia }(32 \%, 11 \%) \\
\text { Fatigue }(28 \%, 18 \%) \\
\text { Contusion }(24 \%, 15 \%) \\
\text { Arthralgia }(22 \%, 16 \%) \\
\text { Atrial Fibrillation }(3 \%, 4 \%) \\
\text { Grade } \geq 3(\mathbf{3}+\mathbf{0}, \mathbf{A}): \\
\text { Neutropenia }(30 \%, 10 \%) \\
\text { Thrombocytopenia (8\%, } \\
5 \%) \\
\text { Anemia }(6 \%, 7 \%)\end{array}$ \\
\hline
\end{tabular}

Abbreviations: AEs, adverse events; TN, treatment-naïv; RR, relapsed/refractory; ORR, overall response rate; PFS, progression-free survival; PRL, partial response with lymphocytosis; DOR, duration of response; $A+O$, acalabrutinib + obinutuzumab cohort; $A$, acalabrutinib monotherapy cohort.

$(\mathrm{n}=8)$. Causality assessment to acalabrutinib therapy was not included in data review. In the same report, treatment discontinuations and dose reductions due to toxicity were rare, with adverse effects of therapy leading to treatment discontinuation and dose reduction in $9 \%$ and $4 \%$ of the patients, respectively. Median time to treatment discontinuation was 7.9 months. With the limit of a cross-trial comparison, the rates of treatment discontinuations and dose reductions in acalabrutinib-treated patients appears to be lower in comparison to ibrutinib. ${ }^{41}$

In the previously mentioned phase III ASCEND trial, grade $3 / 4$ AEs were less frequent in patients treated with acalabrutinib (observed in 70 of 154 patients) compared to patients treated with idelalisib plus rituximab (observed in 101 of 118 patients). ${ }^{29}$ The most common AEs in patients treated with acalabrutinib were neutropenia $(n=24,16 \%)$, anemia $(\mathrm{n}=18,12 \%)$ and pneumonia $(\mathrm{n}=8,5 \%)$.
The recently published data from the phase III ELEVATE-RR trial provide a direct comparison between acalabrutinib and ibrutinib, used in the relapsed/refractory setting. ${ }^{30}$ Overall, grade $3 / 4$ AEs were observed in $68.8 \%$ and $74.9 \%$ of the patients treated with acalabrutinib and ibrutinib, respectively, and AEs led to treatment discontinuation in $14.7 \%$ and $21.3 \%$ of the patients, respectively. Grade 3/4 infections occurred at comparable rates $(30.8 \%$ in acalabrutinib-treated patients vs $30 \%$ in ibrutinib-treated patients). Among the AEs with a significantly different incidence in the two cohorts, patients treated with ibrutinib showed a higher incidence of diarrhea (any grade, $46 \%$ vs $34.6 \%$; grade $3 / 4,4.9 \%$ vs $1.1 \%$ ), arthralgia (any grade, $22.8 \%$ vs $15.8 \%$; grade $3 / 4,0.8 \%$ vs $0 \%$ ), hypertension (any grade, $22.8 \%$ vs $8.6 \%$; grade $3 / 4,8.7 \%$ vs $4.1 \%$ ) and atrial fibrillation (any grade, $15.6 \%$ vs $9 \%$; grade $3 / 4,3.4 \%$ vs $4.5 \%$ ). Patients treated with acalabrutinib showed a 
higher incidence of headache (any grade, $34.6 \%$ vs $20.2 \%$; grade $3 / 4,1.5 \%$ vs $0 \%$ ) and cough (any grade, $28.9 \%$ vs $21.3 \%$; grade $3 / 4,0.8 \%$ vs $0.4 \%$ ).

While there have been limited head-to-head clinical trials of BTK inhibitors with other oral targeted agents, specifically venetoclax, a recent network meta-analysis of trials of frontline CLL therapies compared efficacy and safety of three combinations: acalabrutinib plus obinutuzumab, ibrutinib plus obinutuzumab and venetoclax plus obinutuzumab. $^{42}$ With the limitations of a meta-analysis report, the findings revealed no statistically significant difference in $\mathrm{AE}$ rates among the three treatment cohorts. As a comparison, a comprehensive pooled safety analysis of venetoclax monotherapy in 350 patients with median exposure to therapy of 16 months found that the most common AEs of any grade were diarrhea (41\%), neutropenia $(40 \%)$, nausea $(39 \%)$, anemia $(31 \%)$, fatigue $(28 \%)$ and upper respiratory tract infection $(25 \%){ }^{43}$ The most common grade 3/4 AEs were neutropenia (37\%), anemia (17\%) and thrombocytopenia (14\%). Of the AEs, 10\% led to treatment discontinuation, which is comparable to treatment discontinuation rates reported in patients treated with acalabrutinib. While there are no significant differences in frequency of AEs among treatment agents, a notable distinction is the higher incidence of neutropenia in venetoclax as compared to that seen in acalabrutinib.

\section{Description of Cardiovascular and Bleeding Events Observed in Clinical Trials with BTK Inhibitors}

While they are not the most common side effects or AEs associated with BTK inhibitor therapy, it is paramount to understand the potential for the cardiovascular and bleeding events observed with this drug class. Atrial fibrillation has been reported as a toxicity that is class-specific for BTK inhibitors. The mechanism is not completely understood; however, the increased frequency of atrial fibrillation during BTK inhibitor therapy is thought to be mediated by the inhibition of the PI3K-Akt pathway as there is a cross-talk between BTK and Tec and the PI3KAkt pathway, and a reduced PI3K signaling can lead to increased susceptibility to atrial fibrillation. ${ }^{44-46}$ The rate of atrial fibrillation varies according to the type of trial and length of follow-up. A systemic review of ibrutinib-associated atrial fibrillation from 16 studies with a median follow-up of 18.32 months found that $8.15 \%$ of the patients (190 of 2166) developed atrial fibrillation while on therapy. ${ }^{9}$ The final analysis of the RESONATE trial found an incidence of any grade atrial fibrillation of $12 \%$ in the patients randomized to ibrutinib (24 of 195), with follow-up as long as 71 months. $^{3}$

In current follow-up data, the incidence of atrial fibrillation in patients treated with acalabrutinib appears lower. This is possibly a consequence of the more selective kinase-inhibitory spectrum of this agent. A pooled analysis of 610 patients on acalabrutinib revealed atrial fibrillation episodes of any grade at a rate of $2.3 \% .{ }^{47}$ In patients with relapsed/refractory CLL receiving therapy with acalabrutinib in the ASCEND trial, there was a $6 \%$ frequency of atrial fibrillation ( 9 of 154), reported at a median follow-up of 22 months. ${ }^{29}$ In patients receiving frontline therapy with acalabrutinib in the ELEVATE-TN trial, the incidence of atrial fibrillation was 4\% (7 of 179) in patients receiving treatment with acalabrutinib as monotherapy, 3\% (6 of 178) in patients receiving acalabrutinib-obinutuzumab and $1 \%$ ( 1 of 169$)$ in patients receiving obinutuzumabchlorambucil, at a median follow-up of 28.3 months. ${ }^{34}$

Another cardiovascular toxicity observed in patients receiving BTK inhibitors is the developing of hypertension, or the worsening of a pre-existing hypertension. A retrospective analysis at the Ohio State University that evaluated patients with lymphoid malignancies treated with ibrutinib found that $78 \%$ developed new or worsened hypertension as defined by systolic blood pressure $>130 \mathrm{mmHg}$. ${ }^{48}$ For the patients that were without hypertension at start of ibrutinib treatment $(\mathrm{n}=215), 71.6 \%$ developed new hypertension, with a mean increase in systolic blood pressure of 13.4 $\mathrm{mmHg}$. Similarly, a recent report of 8-year long-term follow-up of ibrutinib on the pivotal PCYC-1102 study found that $28 \%$ of 132 patients had grade 3 or higher hypertension, defined as blood pressure $>160 / 100{ }^{41}$

A pooled analysis of 762 patients across four clinical trials with acalabrutinib monotherapy revealed hypertension in $9 \%$ of the patients, with the majority having prior history of hypertension ( $69 \%$ of patients). ${ }^{49}$ A recent report on ACE-CL-001 trial assessing long-term acalabrutinib safety and tolerability data in treatment-naïve patients found that hypertension of any grade was reported in $22 \%$ of the patients, and half of these events were grade $>3$ or higher. $^{50}$

Bleeding/hemorrhage is one other potential complication of interest to be conscientious of when patients are on BTK inhibitor therapy. Most bleeding events are minor grade and do not require dose adjustments or discontinuation of therapy. In a 3-year follow-up of patients receiving 
single-agent ibrutinib, $61 \%$ of the patients had a bleeding AE. ${ }^{51}$ Of these, $48 \%$ were grade $1,5 \%$ grade $2,7 \%$ grade 3 and $1 \%$ grade 5 . The most commonly reported terms that contributed to bleeding rate were contusion and petechiae. Bleeding/hemorrhage events are similarly seen during therapy with acalabrutinib. The recently reported pooled analysis of 1040 patients on acalabrutinib found $46 \%$ of AEs related to hemorrhage events and included contusion $(n=226,22 \%)$, petechiae $(n=11,11 \%)$, epistaxis $(n=73$, $7 \%)$, ecchymosis $(n=66,6 \%)$ and increased tendency to bruise $(\mathrm{n}=55,5 \%) .{ }^{40}$ Of the reported bleeding events, only $3 \%$ were grade 3 or higher. Of the major hemorrhage events, $43 \%$ occurred in patients on concurrent anticoagulation or antiplatelet therapy.

\section{Clinical Management of Patients on Acalabrutinib}

In the shift to oral targeted therapies for CLL, patient tolerability and compliance is crucial to obtaining efficacy and maximum benefit of therapy. Thus, appropriate understanding of clinical management of potential AEs of acalabrutinib is important. A thorough history and understanding of a patient's comorbidities and risk factors for cardiovascular disease is necessary. This assessment may include a baseline electrocardiogram and echocardiogram for patients with cardiovascular risk factors. Patients should be counseled on interventions that help mitigate risk of atrial fibrillation including smoking cessation, alcohol and caffeine intake reduction, weight loss in setting of obesity, logging of blood pressure and controlling blood sugar. Encouraging a regular exercise routine in accordance with the American Heart Association (150 minutes of moderate aerobic activity per week or 75 minutes of vigorous aerobic activity per week) may be a reference point for exercise goals. ${ }^{52}$

If atrial fibrillation occurs during acalabrutinib therapy, consultation with a cardiologist, or an onco-cardiologist if available, is recommended to help formulate management plan utilizing $\mathrm{CHA}_{2} \mathrm{DS}_{2}$-VASc scores for risk of stroke. Acalabrutinib therapy may need to be continued or interrupted depending on the severity of atrial fibrillation and risk for cardiovascular event. For rate and rhythm control, it is important to consider that CYP3A4 inhibitors (ie, diltiazem, verapamil and amiodarone) interact with acalabrutinib and can increase its serum concentration. Thus, betablockers are preferred agents for rate/rhythm control. Given the antiplatelet effects of BTK inhibitors, recommendation is to reserve use of anticoagulation for setting of higher risk of stroke $\left(\mathrm{CHA}_{2} \mathrm{DS}_{2}\right.$-VASc $\left.\geq 2\right)$ as addition of anticoagulant increases the risk of adverse bleeding events. Recommended agents include low-dose apixaban at $2.5 \mathrm{mg}$ twice daily or enoxaparin at standard dosing. In patients with high $\mathrm{CHA}_{2} \mathrm{DS}_{2}$-VASc scores, an in-depth discussion of the risks and benefits of continuation of treatment with acalabrutinib needs to be conducted by a multidisciplinary team.

For optimal management of the increased bleeding risk associated with acalabrutinib, it is vital to hold acalabrutinib therapy in the setting of procedural interventions. For minor procedures, acalabrutinib should be held three days before and after. For major surgical procedures, this window should increase to 7 days before and after. In the setting of any new or recurrent bleeding, acalabrutinib needs to be held until hemostasis has been achieved. Following major spontaneous bleeding events in the absence of other risk factors, assessment needs to be done if acalabrutinib therapy is safe to resume or if switch to alternative class of therapeutic agents is appropriate. Patients should be advised to avoid non-essential overthe-counter medications that also carry bleeding risk, including aspirin, non-steroidal anti-inflammatory medications and fish oils.

In addition to the management of the potentially more serious AEs, there are supportive care considerations to have for the more common, albeit less severe toxicities. For the most common AE of headache, patient reassurance is key as studies revealed these events to be more selflimited in the early weeks to months of treatment. Patients can be advised to take caffeine or acetaminophen if needed. For arthralgias, there are limited data supporting specific pharmacologic intervention. Steroids and non-steroidal anti-inflammatory medications should be used with caution due to immunosuppressive and bleeding risks without consistent improvement in case studies where they have been utilized for this purpose. Due to potential for increases in serum uric acid levels as cells lyse with therapy, it is appropriate for patients to continue uric acid reducing medications to prevent complications of gout, while white counts remain elevated at the beginning of therapy. In addition, dose reduction can be considered in patients with arthralgias. ${ }^{53}$ For diarrhea and gastro-intestinal distress, as symptoms are often grade 1 or 2 , supportive care with avoidance of dietary triggers and use of antimotility agents is appropriate. During severe episodes, a short treatment hold and then retrial is reasonable. For 
dermatologic eruptions, if not severe, supportive care with topical steroids is effective. Overall, patient understanding and proactive measures for prevention and management of side effects are key for mitigating potential complications during therapy.

Besides the development of AEs, another potential factor that may hamper patients' compliance is the twice daily administration schedule of acalabrutinib. However, it has been shown that patient-specific clinical factors, rather than regimen-specific factors, mainly predict the adherence to oral targeted therapy in the real-world setting. ${ }^{54}$ Finally, it is worth mentioning that, unlike ibrutinib, for patients taking acalabrutinib, the co-administration with proton pump inhibitors needs to be avoided, in order to maintain the correct absorption of the drug.

\section{Infectious Risk in Patients Treated with Acalabrutinib}

The tumor microenvironment of CLL is known to create aberrations in innate and adaptive immune responses, resulting in an increased risk of infection. ${ }^{55,56}$ Infection prevention and management are, therefore, important aspects of caring for a CLL patient population, and thus examination on acalabrutinib's effect on immunosuppression and infection risk over time is warranted. In an updated review of phase II trial of acalabrutinib monotherapy in relapsed/refractory CLL, of the grade 3 or higher AEs reported, neutropenia occurred at frequency of $14 \%$ and pneumonia at $11 \%{ }^{28}$

It is important to consider the immune suppression that BTK inhibitors may inadvertently pose through their activity profile. An in vitro study designed to assess the BTK inhibition on immune response to fungal infection found that exposure to ibrutinib and acalabrutinib reduced signaling pathways activated by Aspergillus fumigatus determining an exacerbation of an immunosuppressive signature, a reduction of phagocytosis and a significant deficit in the secretion of inflammatory cytokines either in macrophages and monocytes. ${ }^{57}$ An additional study assessing the immune effects of BTK inhibitors found that ibrutinib targets BTK expressed by CLL-associated monocyte/macrophage cell population (also known as nurse-like cells, NLC) accentuating their immunosuppressive profile through up-regulation of M2 polarization markers and impairing the phagocytic activity. ${ }^{58}$ In a retrospective analysis of patients receiving ibrutinib for CLL or mantle cell lymphoma at Memorial Sloan
Kettering, an incidence of invasive fungal pneumonia at $37.2 \%$ was found. ${ }^{59}$ Another study following patients on single-agent ibrutinib report 5 cases in 96 patients of Pneumocystis jirovecii pneumonia (PJP). ${ }^{60}$ While it is difficult to determine the exact incidence of fungal infections in the CLL population, cumulative data estimate that the risk of fungal infection is between $0.5 \%$ and $18 \%$ taking into account age, length of CLL diagnosis, and number of prior therapies. ${ }^{61}$

Given the more recent advent of acalabrutinib versus ibrutinib, studies examining fungal and other infections in this treatment population remain limited, and further examination of the more selective inhibitors is needed to better understand if the risks of infections might change among different compounds. Overall, there are insufficient data - to date - to strongly support the routine use of antimicrobial prophylaxis in patients with CLL treated with acalabrutinib and other BTK inhibitors, and practices tend to vary across institutions. According to the 2021 NCCN guidelines, the recommendation is to consider PJP and Varicella zoster virus prophylaxis, and to monitor for fungal infections. Efforts have been made to determine factors that might increase the risk of infections in patients with CLL treated with BTK inhibitors, ${ }^{62}$ but more tools are needed to better stratify the risk of these patients in order to consider additional precautions or prophylaxis for those who are at high risk. Also, the concomitant administration of an anti-CD20 monoclonal antibody needs to be considered when assessing the infectious risk of the patient.

Another aspect to consider is the effect of therapy on immunoglobulin levels. A study reviewing two phase II clinical trials of ibrutinib and acalabrutinib found that median serum IgA levels increased by $41 \%$ and $42 \% 6$ months after the initiation of ibrutinib and acalabrutinib therapy, respectively. ${ }^{63}$ Ibrutinib therapy was previously reported to lead in a slight decrease in serum IgG levels with median reduction of $23 \%$ at 24 months, and no significant difference from pretreatment levels of $\operatorname{IgM}^{64}$ For acalabrutinib, serum IgG and IgM levels did not significantly change during therapy. ${ }^{63}$ Of the acalabrutinib treated cohort, 30 of 41 patients $(73.2 \%)$ developed a total of 66 infections, including $58(87.9 \%)$ grade $1-2,7$ $(10.6 \%)$ grade 3 and 1 (1.5\%) grade 4 infections. The rate of infections during the first 6 months of treatment with acalabrutinib was higher (12.5 infections per 100 patient months) compared to that observed after 6 months of treatment (6.1 infections per 100 patient months). While these 
findings continue to emphasize the infection frequency in CLL even with treatment, the lack of significant suppression of immunoglobulin levels during acalabrutinib treatment does not necessitate the need for gamma globulin replacement as part of supportive care outside of standard guidelines.

\section{Mechanisms of Acalabrutinib Resistance}

Ibrutinib-treated patients may develop resistance to the drug, which has been mainly attributed to the selection of a clone carrying mutation of BTK or of its downstream effector PLCG2. ${ }^{65-67}$ The most common resistance-causing BTK mutation consists in a cysteine to serine substitution at position 481 that decreases the binding affinity between ibrutinib and the kinase. The mechanisms of resistance to acalabrutinib have been less extensively investigated; however, it is expected that they would be similar to those reported in patients treated with ibrutinib, given the shared binding of the two molecules to the C481 site. In the 2020 update of the ACE-CL-001 trial, among 9 patients who were longitudinally assessed for acquired mechanisms of treatment resistance, $67 \%$ had a $\mathrm{C} 481$ BTK mutation at the time of progression and no PLCG2 mutations were detected. ${ }^{28}$ More data regarding acalabrutinib-resistance mechanisms are certainly needed, considering that these may be different to those observed with ibrutinib, given the increased selectivity of acalabrutinib and its possible longer BTK occupancy over time due to twice-daily dosing.

\section{Conclusion and Perspectives}

In an emerging landscape of multiple approved treatments for CLL, acalabrutinib has emerged as a viable therapy option. As a second-generation BTK inhibitor, it has shown efficacy and a favorable safety and toxicity profile. Importantly, responses are observed in patients with highrisk markers and in patients who had discontinued treatment with ibrutinib because of intolerance.

As the CLL treatment options expand and we face multiple agents and combinations of various agents approved in both the frontline and refractory settings, it is becoming relevant to create individualized treatment plans. Several important factors should be considered and include What is the age of the patient? What are their cardiovascular risk factors and infectious risks? Does the patient have high-risk markers for eventual resistance to therapy or transformation to high-grade lymphoma? For certain patients, a dual or triple therapy regimen may be appropriate with the goal of reaching a deep response and allow treatment discontinuation. Given the favorable toxicity profile reported in patients with CLL treated with acalabrutinib as monotherapy or in combination with obinutuzumab, several ongoing studies are evaluating acalabrutinib in combination with the $\mathrm{Bcl}-2$ antagonist venetoclax and monoclonal antibodies.

\section{Disclosure}

$\mathrm{CV}$ reports personal fees from Janssen, outside the submitted work. JLG reports personal fees from ASCO for Faculty for ASCO Advantage: CLL program in October 2021 and JADPRO/APSHO for being the lead author for the updated 2021 Education Module on BTK Inhibitors for APSHO (Advanced Practitioner Society for Hematology Oncology), outside the submitted work. AF received research support from Acerta (a member of the AstraZeneca Group). The authors report no other conflicts of interest in this work.

\section{References}

1. SEER cancer statistics. Available from: https://seer.cancer.gov/stat facts/html/clyl.html. Accessed August 17, 2021.

2. Niemann CU, Wiestner A. B-cell receptor signaling as a driver of lymphoma development and evolution. Semin Cancer Biol. 2013;23 (6):410-421. doi:10.1016/j.semcancer.2013.09.001

3. Munir T, Brown JR, O'Brien S, et al. Final analysis from RESONATE: up to six years of follow-up on ibrutinib in patients with previously treated chronic lymphocytic leukemia or small lymphocytic lymphoma. Am J Hematol. 2019;94(12):1353-1363. doi:10.1002/ajh.25 638

4. Burger JA, Barr PM, Robak T, et al. Long-term efficacy and safety of first-line ibrutinib treatment for patients with CLL/SLL: 5 years of follow-up from the phase 3 RESONATE-2 study. Leukemia. 2020;34 (3):787-798. doi:10.1038/s41375-019-0602-x

5. Woyach JA, Ruppert AS, Heerema NA, et al. Ibrutinib regimens versus chemoimmunotherapy in older patients with untreated CLL. $N$ Engl J Med. 2018;379(26):2517-2528. doi:10.1056/NEJMoa181 2836

6. Moreno C, Greil R, Demirkan F, et al. Ibrutinib plus obinutuzumab versus chlorambucil plus obinutuzumab in first-line treatment of chronic lymphocytic leukaemia (iLLUMINATE): a multicentre, randomised, open-label, phase 3 trial. Lancet Oncol. 2019;20(1):43-56. doi:10.1016/S1470-2045(18)30788-5

7. Shanafelt TD, Wang XV, Kay NE, et al. Ibrutinib-rituximab or chemoimmunotherapy for chronic lymphocytic leukemia. $N$ Engl J Med. 2019;381(5):432-443. doi:10.1056/NEJMoa1817073

8. Caron F, Leong DP, Hillis C, Fraser G, Siegal D. Current understanding of bleeding with ibrutinib use: a systematic review and metaanalysis. Blood Adv. 2017;1(12):772-778. doi:10.1182/bloodadvances. 2016001883 
9. Ganatra S, Sharma A, Shah S, et al. Ibrutinib-associated atrial fibrillation. JACC Clin Electrophysiol. 2018;4(12):1491-1500. doi:10.10 16/j.jacep.2018.06.004

10. Caldeira D, Alves D, Costa J, Ferreira JJ, Pinto FJ. Ibrutinib increases the risk of hypertension and atrial fibrillation: systematic review and meta-analysis. PLoS One. 2019;14(2):e0211228. doi:10.1371/journal. pone. 0211228

11. Barr PM, Brown JR, Hillmen P, et al. Impact of ibrutinib dose adherence on therapeutic efficacy in patients with previously treated CLL/SLL. Blood. 2017;129(19):2612-2615. doi:10.1182/blood2016-12-737346

12. Honigberg LA, Smith AM, Sirisawad M, et al. The Bruton tyrosine kinase inhibitor PCI-32765 blocks B-cell activation and is efficacious in models of autoimmune disease and B-cell malignancy. Proc Natl Acad Sci U S A. 2010;107(29):13075-13080. doi:10.1073/pnas.10 04594107

13. Byrd JC, Harrington B, O'Brien S, et al. Acalabrutinib (ACP-196) in relapsed chronic lymphocytic leukemia. $N$ Engl J Med. 2016;374 (4):323-332. doi:10.1056/NEJMoa1509981

14. Herman SEM, Montraveta A, Niemann CU, et al. The Bruton Tyrosine Kinase (BTK) inhibitor acalabrutinib demonstrates potent on-target effects and efficacy in two mouse models of chronic lymphocytic leukemia. Clin Cancer Res. 2017;23(11):2831-2841. doi:10.1158/1078-0432.CCR-16-0463

15. Barf T, Covey T, Izumi R, et al. Acalabrutinib (ACP-196): a covalent Bruton tyrosine kinase inhibitor with a differentiated selectivity and in vivo potency profile. J Pharmacol Exp Ther. 2017;363(2):240252. doi:10.1124/jpet.117.242909

16. Kaptein A, de Bruin G, Emmelot-van Hoek M, et al. Potency and selectivity of BTK Inhibitors in clinical development for B-cell malignancies. Blood. 2018;132(Supplement 1):1871. doi:10.1182/ blood-2018-99-109973

17. Bye AP, Unsworth AJ, Desborough MJ, et al. Severe platelet dysfunction in NHL patients receiving ibrutinib is absent in patients receiving acalabrutinib. Blood $A d v$. 2017;1(26):2610-2623. doi:10. 1182/bloodadvances.2017011999

18. Series J, Garcia C, Levade M, et al. Differences and similarities in the effects of ibrutinib and acalabrutinib on platelet functions. Haematologica. 2019;104(11):2292-2299. doi:10.3324/haematol.20 18.207183

19. Patel V, Balakrishnan K, Bibikova E, et al. Comparison of acalabrutinib, A selective Bruton tyrosine kinase inhibitor, with ibrutinib in chronic lymphocytic leukemia cells. Clin Cancer Res. 2017;23 (14):3734-3743. doi:10.1158/1078-0432.CCR-16-1446

20. Long M, Beckwith K, Do P, et al. Ibrutinib treatment improves T cell number and function in CLL patients. J Clin Invest. 2017;127 (8):3052-3064. doi:10.1172/JCI89756

21. Kohrt HE, Sagiv-Barfi I, Rafiq S, et al. Ibrutinib antagonizes rituximab-dependent NK cell-mediated cytotoxicity. Blood. 2014;123 (12):1957-1960. doi:10.1182/blood-2014-01-547869

22. Da Roit F, Engelberts PJ, Taylor RP, et al. Ibrutinib interferes with the cell-mediated anti-tumor activities of therapeutic CD20 antibodies: implications for combination therapy. Haematologica. 2015;100 (1):77-86. doi:10.3324/haematol.2014.107011

23. Borge M, Almejun MB, Podaza E, et al. Ibrutinib impairs the phagocytosis of rituximab-coated leukemic cells from chronic lymphocytic leukemia patients by human macrophages. Haematologica. 2015;100:e140-e142. doi:10.3324/haematol.2014.119669

24. Golay J, Ubiali G, Introna M. The specific Bruton tyrosine kinase inhibitor acalabrutinib (ACP-196) shows favorable in vitro activity against chronic lymphocytic leukemia B cells with CD20 antibodies. Haematologica. 2017;102(10):e400-e403. doi:10.3324/haematol.2017.169334

25. Patel VK, Lamothe B, Ayres ML, et al. Pharmacodynamics and proteomic analysis of acalabrutinib therapy: similarity of on-target effects to ibrutinib and rationale for combination therapy. Leukemia. 2018;32(4):920-930. doi:10.1038/leu.2017.321
26. Byrd JC, Furman RR, Coutre SE, et al. Targeting BTK with ibrutinib in relapsed chronic lymphocytic leukemia. N Engl J Med. 2013;369 (1):32-42. doi:10.1056/NEJMoa1215637

27. Sun C, Nierman P, Kendall EK, et al. Clinical and biological implications of target occupancy in CLL treated with the BTK inhibitor acalabrutinib. Blood. 2020;136(1):93-105. doi:10.1182/blood.2019003715

28. Byrd JC, Wierda WG, Schuh A, et al. Acalabrutinib monotherapy in patients with relapsed/refractory chronic lymphocytic leukemia: updated phase 2 results. Blood. 2020;135(15):1204-1213. doi:10. 1182/blood.2018884940

29. Ghia P, Pluta A, Wach M, et al. ASCEND: phase III, randomized trial of acalabrutinib versus idelalisib plus rituximab or bendamustine plus rituximab in relapsed or refractory chronic lymphocytic leukemia. $J$ Clin Oncol. 2020;38(25):2849-2861. doi:10.1200/JCO.19.03355

30. Byrd JC, Hillmen P, Ghia P, et al. Acalabrutinib versus ibrutinib in previously treated chronic lymphocytic leukemia: results of the first randomized phase III trial. J Clin Oncol. 2021;39:3441-3452.

31. Burger JA, Sivina M, Jain N, et al. Randomized trial of ibrutinib vs ibrutinib plus rituximab in patients with chronic lymphocytic leukemia. Blood. 2019;133(10):1011-1019. doi:10.1182/blood-2018-10879429

32. Woyach JA, Blachly JS, Rogers KA, et al. Acalabrutinib plus obinutuzumab in treatment-naïve and relapsed/refractory chronic lymphocytic leukemia. Cancer Discov. 2020;10(3):394-405. doi:10.1158/ 2159-8290.CD-19-1130

33. Byrd JC, Woyach JA, Furman RR, et al. Acalabrutinib in treatmentnaive chronic lymphocytic leukemia. Blood. 2021;137(24):33273338. doi:10.1182/blood.2020009617

34. Sharman JP, Egyed M, Jurczak W, et al. Acalabrutinib with or without obinutuzumab versus chlorambucil and obinutuzumab for treatment-naive chronic lymphocytic leukaemia (ELEVATE TN): a randomised, controlled, phase 3 trial. Lancet. 2020;395(102 32):1278-1291. doi:10.1016/S0140-6736(20)30262-2

35. Sharman JP, Egyed M, Jurczak W, et al. Acalabrutinib \pm obinutuzumab versus obinutuzumab + chlorambucil in treatment-naïve chronic lymphocytic leukemia: elevate-TN four-year follow up. J Clin Oncol. 2021;39(15_suppl):7509. doi:10.1200/JCO.2021.39.15_suppl.7509

36. Delgado J, Josephson F, Camarero J, et al. EMA review of acalabrutinib for the treatment of adult patients with chronic lymphocytic leukemia. Oncologist. 2021;26(3):242-249. doi:10.1002/onco.13685

37. Alrawashdh N, Persky DO, McBride A, Sweasy J, Erstad B, Abraham I. Comparative efficacy of first-line treatments of chronic lymphocytic leukemia: network meta-analyses of survival curves. Clin Lymphoma Myeloma Leuk. 2021;21(11):e820-e831. doi:10.10 16/j.clml.2021.06.010

38. Awan FT, Schuh A, Brown JR, et al. Acalabrutinib monotherapy in patients with chronic lymphocytic leukemia who are intolerant to ibrutinib. Blood Adv. 2019;3(9):1553-1562. doi:10.1182/bloodadvances. 2018030007

39. Rogers KA, Thompson PA, Allan JN, et al. Phase 2 study of acalabrutinib in ibrutinib (IBR)-intolerant patients (pts) with relapsed/refractory (R/R) chronic lymphocytic leukemia (CLL). J Clin Oncol. 2019;37 (15_suppl):7530. doi:10.1200/JCO.2019.37.15_suppl.7530

40. Furman RR, Byrd JC, Owen RG, et al. Pooled analysis of safety data from clinical trials evaluating acalabrutinib monotherapy in mature B-cell malignancies. Leukemia. 2021;35:3201-3211. doi:10.1038/ s41375-021-01252-y

41. Byrd JC, Furman RR, Coutre SE, et al. Ibrutinib treatment for firstline and relapsed/refractory chronic lymphocytic leukemia: final analysis of the pivotal phase Ib/II PCYC-1102 study. Clin Cancer Res. 2020;26(15):3918-3927. doi:10.1158/1078-0432.CCR-19-2856

42. Sheng Z, Song S, Yu M, et al. Comparison of acalabrutinib plus obinutuzumab, ibrutinib plus obinutuzumab and venetoclax plus obinutuzumab for untreated CLL: a network meta-analysis. Leuk Lymphoma. 2020;61(14):3432-3439. doi:10.1080/10428194.2020.1811271 
43. Davids MS, Hallek M, Wierda W, et al. Comprehensive safety analysis of venetoclax monotherapy for patients with relapsed/refractory chronic lymphocytic leukemia. Clin Cancer Res. 2018;24 (18):4371-4379. doi:10.1158/1078-0432.CCR-17-3761

44. Qiu Y, Kung HJ. Signaling network of the Btk family kinases. Oncogene. 2000;19(49):5651-5661. doi:10.1038/sj.onc.1203958

45. Pretorius L, Du XJ, Woodcock EA, et al. Reduced phosphoinositide 3-kinase (p110alpha) activation increases the susceptibility to atrial fibrillation. Am J Pathol. 2009;175(3):998-1009. doi:10.2353/ ajpath.2009.090126

46. McMullen JR, Boey EJ, Ooi JY, Seymour JF, Keating MJ, Tam CS. Ibrutinib increases the risk of atrial fibrillation, potentially through inhibition of cardiac PI3K-Akt signaling. Blood. 2014;124(25):3829 3830. doi:10.1182/blood-2014-10-604272

47. Abbas HA, Wierda WG. Acalabrutinib: a selective Bruton tyrosine kinase inhibitor for the treatment of B-cell malignancies. Front Oncol. 2021;11:668162. doi:10.3389/fonc.2021.668162

48. Dickerson T, Wiczer T, Waller A, et al. Hypertension and incident cardiovascular events following ibrutinib initiation. Blood. 2019;134 (22):1919-1928. doi:10.1182/blood.2019000840

49. Brown JR, Byrd JC, Ghia P, et al. Cardiovascular adverse events in patients with chronic lymphocytic leukemia receiving acalabrutinib monotherapy: pooled analysis of 762 patients. Haematologica. 2021. doi:10.3324/haematol.2021.278901

50. Byrd JC, Woyach JA, Furman RR, et al. Acalabrutinib in treatment-naïve chronic lymphocytic leukemia: mature results from phase II study demonstrating durable remissions and long-term tolerability. J Clin Oncol. 2020;38(15_suppl):8024. doi:10.1200/JCO.2020.38.15_suppl.8024

51. Byrd JC, Furman RR, Coutre SE, et al. Three-year follow-up of treatment-naive and previously treated patients with CLL and SLL receiving single-agent ibrutinib. Blood. 2015;125(16):2497-2506. doi:10.1182/blood-2014-10-606038

52. Piercy KL, Troiano RP. Physical activity guidelines for Americans from the US department of health and human services. Circ Cardiovasc Qual Outcomes. 2018;11(11):e005263. doi:10.1161/ CIRCOUTCOMES.118.005263

53. Lipsky A, Lamanna N. Managing toxicities of Bruton tyrosine kinase inhibitors. Hematology. 2020;2020(1):336-345. doi:10.1182/ hematology.2020000118

54. Patel K, Sudhir VS, Kabadi S, et al. Impact of dosing frequency (once daily or twice daily) on patient adherence to oral targeted therapies for hematologic malignancies: a retrospective cohort study among managed care enrollees. J Oncol Pharm Pract. 2019;25 (8):1897-1906. doi:10.1177/1078155219827637

55. Griggio V, Perutelli F, Salvetti C, et al. Immune dysfunctions and immune-based therapeutic interventions in chronic lymphocytic leukemia. Front Immunol. 2020;11:594556. doi:10.3389/fimmu.2020.594556
56. Vitale C, Boccellato E, Comba L, et al. Impact of immune parameters and immune dysfunctions on the prognosis of patients with chronic lymphocytic leukemia. Cancers. 2021;13(15):3856. doi:10.3390/ cancers 13153856

57. Fiorcari S, Maffei R, Vallerini D, et al. BTK inhibition impairs the innate response against fungal infection in patients with chronic lymphocytic leukemia. Front Immunol. 2020;11:2158. doi:10.3389/ fimmu.2020.02158

58. Fiorcari S, Maffei R, Audrito V, et al. Ibrutinib modifies the function of monocyte/macrophage population in chronic lymphocytic leukemia. Oncotarget. 2016;7(40):65968-65981. doi:10.18632/oncotarget. 11782

59. Varughese T, Taur Y, Cohen N, et al. Serious infections in patients receiving ibrutinib for treatment of lymphoid cancer. Clin Infect Dis. 2018;67(5):687-692. doi:10.1093/cid/ciy175

60. Ahn IE, Jerussi T, Farooqui M, Tian X, Wiestner A, Gea-Banacloche J. Atypical pneumocystis jirovecii pneumonia in previously untreated patients with CLL on single-agent ibrutinib. Blood. 2016;128 (15):1940-1943. doi:10.1182/blood-2016-06-722991

61. Facchinelli D, Marchesini G, Nadali G, Pagano L. Invasive fungal infections in patients with chronic lymphoproliferative disorders in the era of target drugs. Mediterr J Hematol Infect Dis. 2018;10(1): e2018063. doi:10.4084/mjhid.2018.063

62. Mauro FR, Giannarelli D, Visentin A, et al. Prognostic impact and risk factors of infections in patients with chronic lymphocytic leukemia treated with ibrutinib. Cancers. 2021;13(13):3240. doi:10.3390/ cancers13133240

63. Pleyer C, Sun C, Desai S, et al. Reconstitution of humoral immunity and decreased risk of infections in patients with chronic lymphocytic leukemia treated with Bruton tyrosine kinase inhibitors. Leuk Lymphoma. 2020;61(10):2375-2382. doi:10.1080/10428194.2020. 1772477

64. Sun C, Tian X, Lee YS, et al. Partial reconstitution of humoral immunity and fewer infections in patients with chronic lymphocytic leukemia treated with ibrutinib. Blood. 2015;126(19):2213-2219. doi:10.1182/blood-2015-04-639203

65. Furman RR, Cheng S, Lu P, et al. Ibrutinib resistance in chronic lymphocytic leukemia. $N$ Engl J Med. 2014;370(24):2352-2354. doi:10.1056/NEJMc1402716

66. Woyach JA, Ruppert AS, Guinn D, et al. BTK(C481S)-mediated resistance to ibrutinib in chronic lymphocytic leukemia. J Clin Oncol. 2017;35(13):1437-1443. doi:10.1200/JCO.2016.70.2282

67. Ahn IE, Underbayev C, Albitar A, et al. Clonal evolution leading to ibrutinib resistance in chronic lymphocytic leukemia. Blood. 2017;129(11):1469-1479. doi:10.1182/blood-2016-06-719294
OncoTargets and Therapy

\section{Publish your work in this journal}

OncoTargets and Therapy is an international, peer-reviewed, open access journal focusing on the pathological basis of all cancers, potential targets for therapy and treatment protocols employed to improve the management of cancer patients. The journal also focuses on the impact of management programs and new therapeutic

Submit your manuscript here: https://www.dovepress.com/oncotargets-and-therapy-journa agents and protocols on patient perspectives such as quality of life, adherence and satisfaction. The manuscript management system is completely online and includes a very quick and fair peer-review system, which is all easy to use. Visit http://www.dovepress.com/ testimonials.php to read real quotes from published authors. 